\title{
Comparison of Se and Te clusters produced by ion bombardment
}

\author{
Matgorzata Trzyna
}

Center for Microelectronics and Nanotechnology, Department of Mathematics and Natural Sciences, Rzeszow University, Poland

\begin{abstract}
Nanostructures based on tellurium and selenium are materials used as components for the manufacturing topological insulators. Therefore it is crucial to precisely characterize these materials. In this work the emission of selenium and tellurium cluster ions, sputtered by $\mathrm{Bi}^{+}$primary ion guns, was investigated by using Time-of-Flight Secondary Ion Mass Spectrometry (TOF SIMS). It has been found that $\mathrm{Bi}_{\mathrm{x}} \mathrm{Te}_{\mathrm{x}}$ and $\mathrm{Bi}_{\mathrm{x}} \mathrm{Se}_{\mathrm{x}}$ clusters appear in addition to $\mathrm{Se}_{\mathrm{x}}$ and $\mathrm{Te}_{\mathrm{x}}$ clusters in the mass range up to $\sim 1300 \mathrm{~m} / \mathrm{z}$. Local maxima or minima (magic numbers) are observed in the ion intensity versus a number of atoms per cluster for both positive and negative ions spectra for all types of clusters and primary ions used. These extrema can be attributed to different yield and stability of certain clusters but also to fragmentation of highmass clusters.
\end{abstract}

\section{Introduction}

Recently, the subject of topological insulators have attracted great attention in solid state physics. Most popular topological structures are based on elements of groups IV-VI and III-VI. Understanding properties of these compounds is crucial for their further development $[1,2]$.

One of them is clusters formation process on the surface which influences the properties of the whole material. Cluster ions are frequently observed to be emitted from a variety of samples exposed to ion beam bombardment. The formation mechanism of these cluster ions is important if they are to be utilized to determine the structure of solid surfaces [3].

The clusters are made by different methods, however the most recent studies focus on the clusters obtained during material exposure to different primary ions. We have used the TOF SIMS as an analytical tool to measure species and clusters. Studies of the whole secondary ion spectrum allow to understand in greater depth how secondary ions are formed, which is of exceptional importance to the whole field of secondary ion mass spectrometry [4]. The purpose of this paper is to investigate the formation of Se and Te cluster ions using $\mathrm{Bi}^{+}$primary ions.

\section{Materials And Methods}

Two materials were chosen for the investigation: Te single-crystal ingots grown by the Czochralski method and the black allotrope of Se obtained by a vacuum distillation method at the Institute of Non-Ferrous Metals, Skawina, Poland. Both with the level of residual metallic impurities corresponding to the $4.5-5 \mathrm{~N}$ standard. The initial samples were obtained by mechanical cleaving under ambient atmospheric conditions, and their surface was covered by native oxide.

Positive and negative SIMS measurements were performed with a TOF-SIMS V instrument (ION-TOF $\mathrm{GmbH}$, Muenster, Germany), using $30 \mathrm{keV} \mathrm{Bi}{ }^{+}$primary ion beams. Secondary ion mass spectra were obtained using a cycle time of $120 \mu$ s to obtain the mass range up to $\sim 1300 \mathrm{~m} / \mathrm{z}$. This allowed detecting the $\mathrm{Te}_{\mathrm{x}}$ and $\mathrm{Se}_{\mathrm{x}}$ clusters with $\mathrm{x} \leq 10$. We used primary ion current $<1.5$ $\mathrm{pA}$ at the crater bottom $100 \times 100 \mu \mathrm{m}^{2}$. The total fluence was less than $5 \times 10^{12} \mathrm{Bi}^{+} \mathrm{cm}^{-2}$ ensuring static conditions and the changes at the spectra after the second measurement in the same place were comparable with the reproducibility of the measurements.

The mode with constant primary ion current (DC mode for the TOF-SIMS V, ion current $40 \mathrm{pA}$, the crater bottom $100 \times 100 \mu \mathrm{m}^{2}$ ) were used to remove surface contaminants. SurfaceLab software $(6.4$ version $)$ of $I O N-T O F$ GmbH was used to manipulate the mass spectra. All spectra were calibrated to at least 5 peaks. 

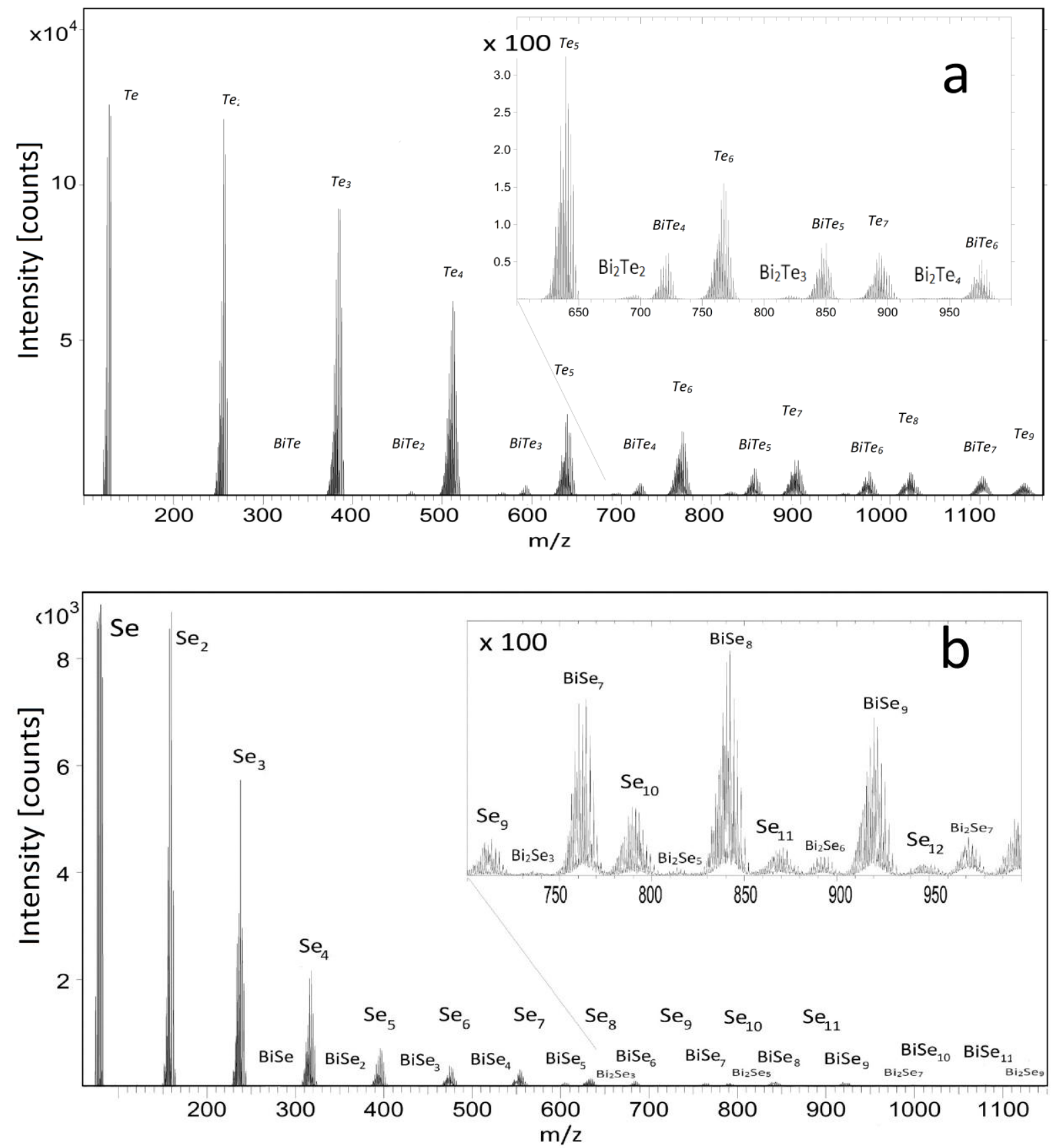

Fig.1.Negative ion mass spectra from Te and Se surfaces which were pre-cleaned with a $\mathrm{Bi}^{+}$at the $\mathrm{DC}$ mode: (a) tellurium (b) selenium.

\section{Results}

Figure 1 illustrates typical features of negative ion mass spectra from Te and Se surfaces which were pre-cleaned with $\mathrm{Bi}^{+}$at the $\mathrm{DC}$ mode.

Both spectra contain three sequences of fragments with a typical Te or Se isotopic distribution. The sequences are shifted towards each other by $209 \mathrm{~m} / \mathrm{z}$, i.e. Bi atom mass. The first sequence is generated by $\mathrm{Te}(\mathrm{Se})_{\mathrm{x}}$ clusters, while the second and the third ones result from $\mathrm{BiTe}(\mathrm{Se})_{\mathrm{x}}$ and $\mathrm{Bi}_{2} \mathrm{Te}(\mathrm{Se})_{\mathrm{x}}$ correspondingly. It is seen in Figure 1 that the mass spectra of secondary ions for both materials are comparable.
At the same time they are very different from the mass spectra of Se and Te clusters produced by the inert gas condensation technique [5] or laser ablation [6].

J. Becker and A. Benamar explain the completely diverse mass spectra of $\mathrm{Se}$ and $\mathrm{Te}$ by the different crystalic structures of these materials. Small cycling modules play a great role in selenium. In tellurium, these periodic structures do not exist and large clusters are easily obtained in nucleation conditions where only small selenium clusters are present $[5,7]$. 

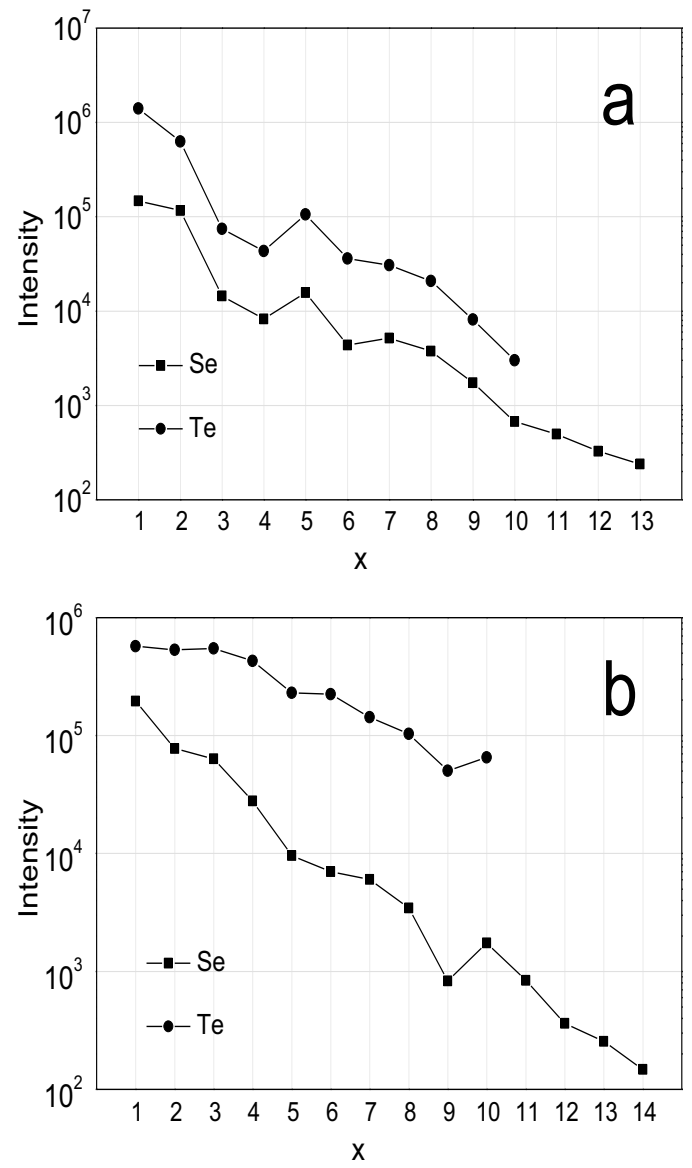

Fig. 2. The comparison of $\mathrm{Te}_{\mathrm{x}}$ and $\mathrm{Se}_{\mathrm{x}}$ clusters peaks intensity as function of the number $\mathrm{x}$ of atoms in the cluster for the sputtering by $30 \mathrm{keV} \mathrm{Bi}^{+}$primary ion beam. (a) positive ions, (b) negative ions.

And we must note that $\mathrm{Se}_{\mathrm{x}}$ and $\mathrm{Te}_{\mathrm{x}}$ clusters distribution is similar. (Fig. 2.) If we ignore curve extremes, we can see that for both positive and negative ions the general trend follows the power law in a good agreement with the results obtained for different semiconductors at sputtering by different primary ions $\left(\mathrm{Ga}^{+}[8], \mathrm{Au}^{+}, \mathrm{Au}^{-}\right.$, $\mathrm{Al}^{-}[4], \mathrm{Ar}^{+}, \mathrm{Cs}^{+}, \mathrm{C}^{+} 0^{+}$[9] etc.).

This permits to suppose that in case of sputtering the mechanism of cluster formation is less dependent on the crystal structure of the starting material.

At the same time for all Se and Te clusters obtained by different methods, the maximum at $\mathrm{x}=5$ is similar to our observed results. (Fig. 2.)

This maxima was also observed by J. Becker at al. They present the first mass-selected ultra- violet photoionphotoelectron coincidence spectra of Se-clusters up to $\mathrm{Se}_{25}$ and of $\mathrm{Te}_{6} \mathrm{Te}$-clusters. Figure 3 presents these mass-spectra of Se- and Te-clusters, which was generated during the adiabatic expansion of molecular beam [5].

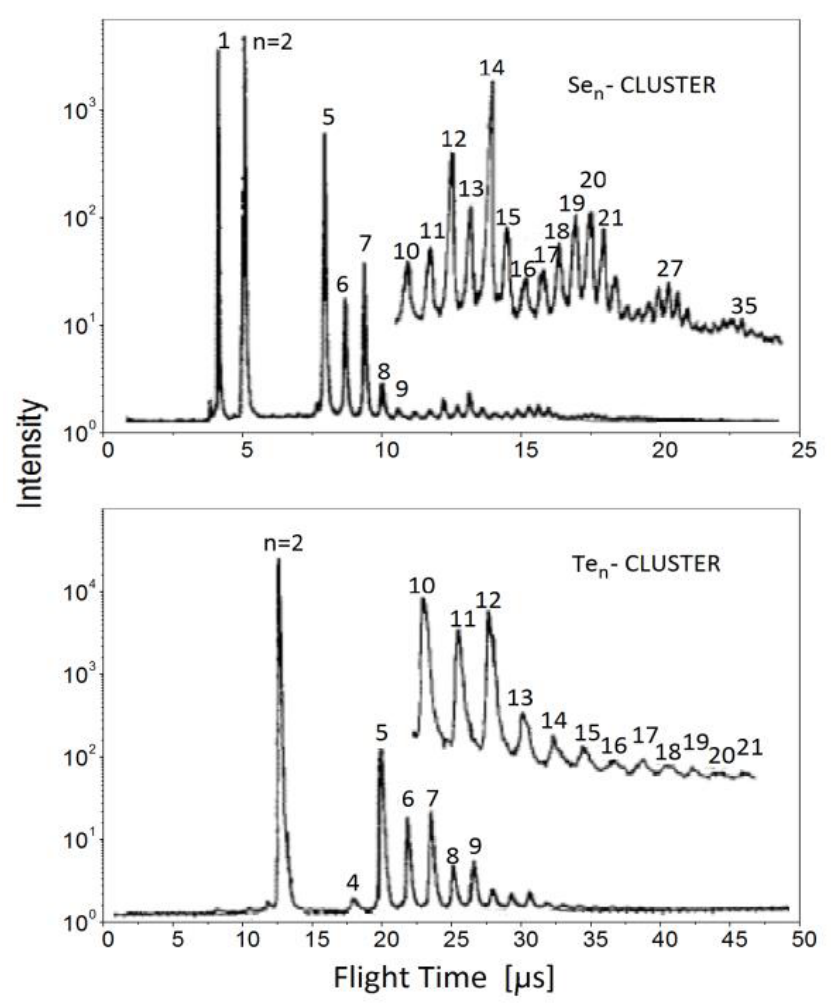

Fig.3.Typical mass-spectra of selenium- and tellurium-clusters obtained by ultraviolet photoion-photoelectron method [5].

Similar results were also obtained by A.K. Hearley et al. The three-dimensional EDLDI method revealed the maximum for clusters formed by the laser ablation of solid samples of some elements (phosphorus, sulfur, selenium, tellurium and their mixtures) [6].

The authors using laser desorption/ionization mass spectrometry method showed the energy dependence of positive and negative ions which are formed by extension to other substances. The results prove existing the maximum of clusters, which are characteristic for the investigated materials.

B.C. Pan theoretically investigated the geometric structures and energetic of positive and neutral small tellurium clusters $\mathrm{Te}_{\mathrm{x}}(\mathrm{x} \leq 8)$, and also showed that the intensity maximum of $\mathrm{Te}_{5}$ in the mass spectra is tied to the maximum at $\mathrm{x}=5$ at the lowest dissociation energy curve of $\mathrm{Te}_{\mathrm{x}}[10]$. 

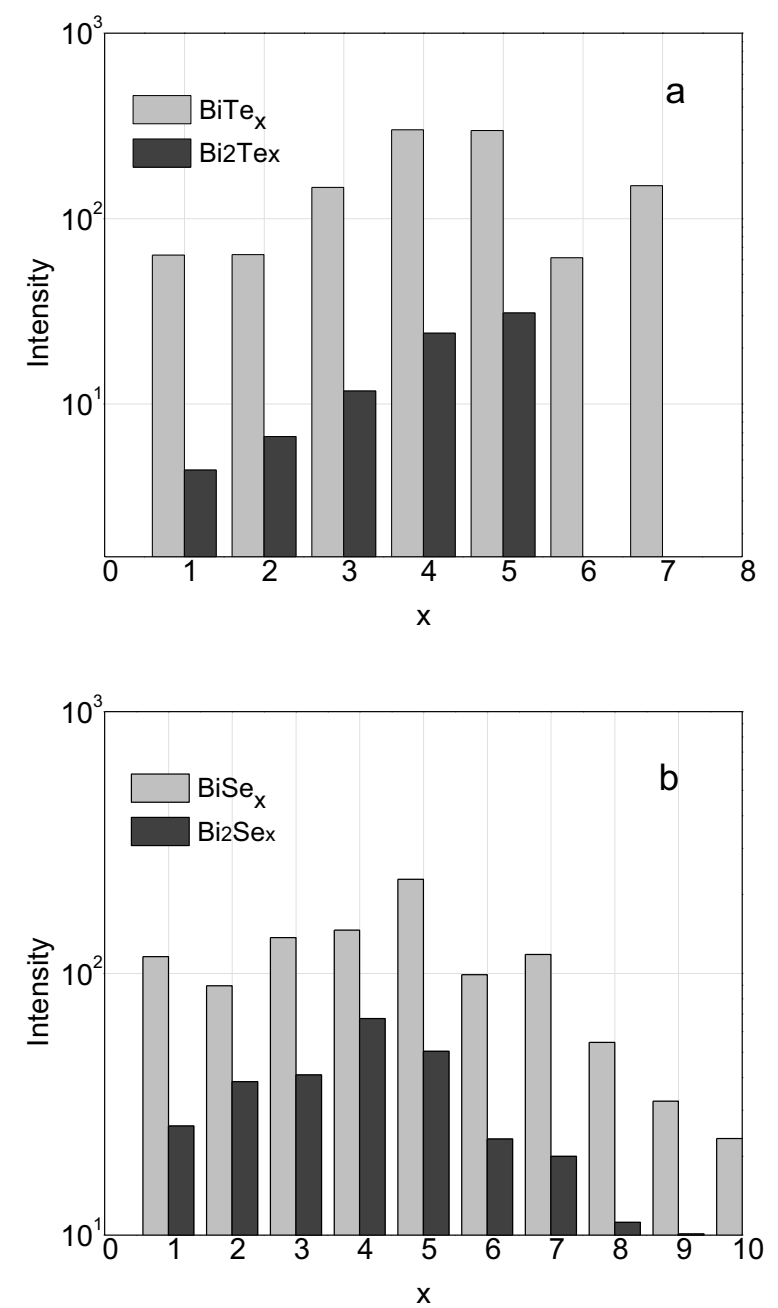

Fig.4. Comparison of peaks intensity of clusters Bi-Se and BiTe ions as function of the number $x$ of atoms in the cluster for positive ions

Figure 4 indicates thats in addition to chalcogen clusters in mass spectra the chalcogen-bismuuth clusters can be seen. As it was demonstrated by Li and Hirokawa, the appearance of such clusters is characteristic for elements forming by atoms of a primary beam of the chemical compound or solid solution. In this case, apart from the sputtering and ionization, the alloying of surface atoms with $\mathrm{Bi}$ atoms takes place [8]. Though many Bi-Se and $\mathrm{Bi}$-Te compounds are possible, we also reveal $\mathrm{Bi}_{2} \mathrm{Se}_{3}$ and $\mathrm{Bi}_{2} \mathrm{Te}_{3}$ as stoichiometric phase in these sequences. But on the dependences of intensity ions of $\mathrm{Bi}_{2} \mathrm{Se}_{\mathrm{x}}$ and $\mathrm{Bi}_{2} \mathrm{Te}_{\mathrm{x}}$ as function of the number $\mathrm{x}$ of atoms in the cluster there is no maximum for $\mathrm{Bi}_{2} \mathrm{Se}_{3}$ and $\mathrm{Bi}_{2} \mathrm{Te}_{3}$. It suggests that during the process of creating the cluster the stoichiometric complex does not appear. (Fig. 4.) A more complete explanation of above dependences requires theoretical calculations similar to those performed for many monoatomic and polyatomic clusters of metals and semiconductors.

\section{Conclusions}

To sum up, the general view of selenium and tellurium mass-spectra is similar. Obtained clusters are comparable with the clusters obtained by different ion bombardment. However local intensity of clusters is specific for Te and Se.

The maximum corresponding to $\mathrm{x}=5$ at the ion spectra Te and Se can be explained theoretically. It may indicate the greater stability of cluster structure with $x=5$ in the these chalcogens.

The appearance of Bi-Se clusters is characteristic for elements forming with atoms a primary beam of the chemical compound. In this case, apart from the sputtering and ionization, the alloying of surface atoms with $\mathrm{Bi}$ atoms takes place.

Interactions between sputtering species and inorganic compounds involve many complicating effects. They may alter the secondary ion yield of matrix atoms and create new peaks that cause unexpected mass interferences.

Besides Bi-Se and Bi-Te clusters may find application as the building blocks for bismuth based on nanomaterials and topological insulators.

\section{References}

1. H. Zhang, C.-X. Liu, X.-L. Qi, X. Dai, Z. Fang, S.-C. Zhang, Nat. Phys. 5, 438-442 (2009)

2. S. Lin, W. Li, Z. Chen, J. Shen, B. Ge, Y. Pei, Nat. Commun. 7, 10287 (2016)

3. B.J.G. N. Winograd, K.E. Foley, Physic Lett. 73, 253-255 (1979)

4. M.P. Seah, F.M. Green, I.S. Gilmore, J. Am. Soc. Mass Spectrom. 21, 370-377 (2010)

5. J. Becker, K. Rademann, F. Hensel, Atom Mol. Clust. 235, 233-235 (1991)

6. A.K. Hearley, B.F.G. Johnson, J.S. McIndoe, D.G. Tuck, Inorganica Chim. Acta. 334, 105112 (2002)

7. A. Benamar, D.I. Rayane, P. Melinon, B. Tribollet, M. Broyer, Atom Mol. Clust. 239 237-239 (1991)

8. Z. Li, K. Hirokawa, Appl. Surf. Sci. 220, 136153 (2003)

9. R.E. Goacher, H. Luo, J.A. Gardella, Anal. Chem. 80, 3261-3269 (2008)

10. B.C. Pan, Phys. Rev. B. 65, 85407 (2002). 